\title{
Acral Lentiginous Melanoma
}

National Cancer Institute

\section{Source}

National Cancer Institute. Acral Lentiginous Melanoma. NCI Thesaurus. Code C4022.

A form of melanoma occurring most often on the plantar, palmar, subungual, and periungual skin. It presents as a pigmented macular lesion with irregular borders.

Morphologically, it consists of atypical spindled and dendritic melanocytes. The epidermis is often hyperplastic and there is pagetoid infiltration of the epidermis by anaplastic cells. 